\title{
An improved weighting method with multibounds formulation and convex programming for multicriteria structural optimization
}

\author{
W. H. Zhang ${ }^{1, *, \dagger}$, M. Domaszewski ${ }^{2}$ and C. Fleury ${ }^{3}$ \\ ${ }^{1}$ Sino-French Laboratory of Concurrent Engineering, Department of Aircraft Manufacturing Engineering, \\ Northwestern Polytechnical University, 710072 Xi'an, Shaanxi, People's Republic of China \\ ${ }^{2}$ Laboratory of Modelling in Mechanics, University of Technology of Belfort-Montbeliard, \\ 90010 Belfort, France \\ ${ }^{3}$ LTAS-Aerospace Laboratory, Institute of Mechanics, University of Liége, 4000 Liége, Belgium
}

\begin{abstract}
SUMMARY
This paper presents an improved weighting method for multicriteria structural optimization. By introducing artificial design variables, here called as multibounds formulation (MBF), we demonstrate mathematically that the weighting combination of criteria can be transformed into a simplified problem with a linear objective function. This is a unified formulation for one criterion and multicriteria problems. Due to the uncoupling of involved criteria after the transformation, the extension and the adaptation of monotonic approximation-based convex programming methods such as the convex linearization (CONLIN) or the method of moving asymptotes (MMA) are made possible to solve multicriteria problems as efficiently as for one criterion problems. In this work, a multicriteria optimization tool is developed by integrating the multibounds formulation with the CONLIN optimizer and the ABAQUS finite element analysis system. Some numerical examples are taken into account to show the efficiency of this approach. Copyright (c) 2001 John Wiley \& Sons, Ltd.
\end{abstract}

KEY WORDS: multicriteria optimization; weighting method; convex programming; structural design

\section{INTRODUCTION}

Regarding the previous summary work by Eschenauer et al. [1], Stadler [2] and Osyczka [3], it is recognized that multicriteria problems, i.e. multiobjective functions often exist in many practical engineering designs. For example, the structural design traditionally concerning the weight minimization, the stress minimization, the stiffness maximization or the minimization

\footnotetext{
${ }^{*}$ Correspondence to: W. H. Zhang, Sino-French Laboratory of Concurrent Engineering, Department of Aircraft Manufacturing Engineering, Northwestern Polytechnical University, 710072 Xi'an, Shaanxi, People's Republic of China

†E-mail: zhang_weihong@yahoo.com
}

Contract/grant sponsor: Aeronautical Foundation of China, Cheung Kong Scholars Programme; contract/grant number: 00B53005

Copyright (c) 2001 John Wiley \& Sons, Ltd.

Received 13 December 1999 Revised 25 January 2001 
of damped resonance amplitudes in dynamics (see Saravanos and Chamis [4]). Whatever be the nature of these criteria is, they are expected to be simultaneously attained at the optimal design solution.

In contrast with the mono-objective function problem, the multicriteria problem has a set of compromising solutions called Pareto optima. The Pareto optimum means that any improvement of a certain number of criteria at its neighbourhood will deteriorate at least another criterion. Many attempts have been made to solve multicriteria problems. Apart from using set theory approaches, there exist a variety of scalarization approaches such as the weighting method, the trade-off method, the goal attainment method and the min-max formulation. Based on the boundary intersection method of Schy et al. [5], Das and Dennis [6] recently proposed a modified version called the normal boundary intersection method. In addition, Li et al. [7] proposed the approximation of using the hyper-ellipse to generate the Pareto optimal curve in the objective space for bicriteria convex optimization problems. Pietrzak [8] proposed the so-called global overlapping index to identify the Pareto solutions and the closeness of any feasible points to them for two design variable problems. In order to reduce the computing costs of finite element reanalyses and sensitivity calculations, Grandhi and Bharatram [9] developed a heuristic multiobjective compound scaling method which was used to deal with the sizing optimization of truss and plate structures.

Although the weighting method has some intrinsic inconveniences, i.e. the incapacity to capture Pareto optimal points of the non-convex attainable region (see Koski [10]); the uniform discrete weightings often leading to very uneven distributions of Pareto optimal points (see Lin [11]; Das and Denis [6]), this method is still considered as a usual method. The reason perhaps lies in that the formulation is straightforward and the solution of the weighting problem is mathematically ensured to be a Pareto optimum. Theoretically, this method proceeds by replacing the vector objective function with a scalar and linear weighting summation. By changing the values of weighting coefficients, a sequence of scalar problems will be defined whose solutions constitute the Pareto optima. Therefore, the efficient resolution of a considerable number of scalar problems is the key problem for the weighting method to be practically applicable.

For this purpose, the multibounds formulation (MBF) is developed in this paper. It has the advantage of converting the original weighting problem even non-differentiable into a simple form. This form is mathematically proved based on the Kuhn-Tucker optimality condition. It is characterized by a linear objective function and each objective criterion is individually transformed into inequality constraint bounded by the related artificial design variable. As a result, the CONLIN optimizer and other monotonic approximation-based optimization algorithms can be efficiently applied because there is no direct coupling among different conflicting criteria. In this study, sizing optimization of shell structures will be solved by the MBF formulation.

\section{MULTIBOUNDS FORMULATION OF THE WEIGHTING METHOD}

\subsection{The weighting method}

The multicriteria optimization is to find the design vector $x$ of $n$-dimension for the following problem:

$$
\begin{gathered}
\operatorname{Min} \mathbf{f}(x) \\
g_{j}(x) \leqslant 0, \quad j=1, m
\end{gathered}
$$


where $\mathbf{f}(x)=\left[f_{1}(x), f_{2}(x), \ldots, f_{r}(x)\right]^{\mathrm{T}}$ is the vector of objective functions to be minimized and $g_{j}(x)$ designates the $j$ th constraint function. We can easily find that the one criterion problem is a particular case when $r=1$.

The solution that independently minimizes each individual criterion is theoretically an ideal and unattainable optimum point. To get the Pareto optimum set by the weighting method, we have to solve the following scalar problem in which the preference function defined by the linear combination of the scalar objective functions will be minimized:

$$
\begin{gathered}
\operatorname{Min} \sum_{k} w_{k} f_{k}(x) \\
g_{j}(x) \leqslant 0, \quad j=1, m,
\end{gathered}
$$

where the $w_{k}$ 's are positive weighting coefficients satisfying the normalization equality $\sum_{k} w_{k}=1$.

To make discussions easy, suppose that the criteria and constraints are all convex and differentiable functions. The preference function is then a convex combination because of positive weighting coefficients $w_{k}$. Without the loss of generality, we can suppose furthermore that involved weighting coefficients are always positive $w_{k}>0$ because for any $w_{k}=0$, the $k$ th term of $w_{k} f_{k}(x)$ will be excluded from (2). As a result, for given values $w_{k}$, a unique optimal solution of (2) exists mathematically. This solution is dominated by the Kuhn-Tucker (K-T) necessary and sufficient optimality condition as follows:

$$
\begin{aligned}
& \nabla_{x} L(x)=\sum_{k} w_{k} \nabla f_{k}(x)+\sum_{j} \mu_{j} \nabla g_{j}(x)=0 \\
& \mu_{j} g_{j}(x)=0, \quad \mu_{j} \geqslant 0
\end{aligned}
$$

in which $\mu_{j}$ denotes the $j$ th Lagrangian multiplier and the Lagrangian function is defined as

$$
L(x)=\sum_{k} w_{k} f_{k}(x)+\sum_{j} \mu_{j} g_{j}(x)
$$

\subsection{The multibounds formulation}

The proposed MBF method is to introduce additional variables $\beta_{k}$ called artificial or slack variables. Each one will be utilized as an upper bound while each criterion is transformed into the inequality constraint. Problem (2) will become

$$
\begin{gathered}
\operatorname{Min} \sum_{k} w_{k} \beta_{k} \\
f_{k}(x) \leqslant \beta_{k}, \quad k=1, r \\
g_{j}(x) \leqslant 0, \quad j=1, m
\end{gathered}
$$

In this formulation, we have $r$ additional design variables and $r$ additional constraints apart from $m$ original constraints and $n$ design variables. If compared with the one-bound formulation of the min-max statement suggested by Bendsøe et al. [12] and Olhoff [13], the above formulation then corresponds to choose particularly $\beta_{k}=\beta / w_{k}$ with only $\beta$ to be the additional design variable. Therefore, the MBF is also a generalization of the one-bound formulation. In fact, the MBF can also be used to deal with one criterion problems with $r=1$. In this 
case, (5) is simplified as

$$
\begin{gathered}
\operatorname{Min} \beta_{1} \\
f_{1}(x) \leqslant \beta_{1} \\
g_{j}(x) \leqslant 0 \quad j=1, m
\end{gathered}
$$

\subsection{Equivalence between the multibounds formulation and the weighting method}

The above explanations point out that the MBF is a general formulation. The multicriteria problem, the single criterion problem as well as the min-max problem can be handled in a unified way when the computing code is programmed.

Now, demonstrations are made to prove that the solution of $\operatorname{MBF}(5)$ is equivalent to that of the original problem (2). By writing the corresponding Lagrangian function of (5)

$$
L(x, \beta)=\sum_{k} w_{k} \beta_{k}+\sum_{k} \lambda_{k}\left(f_{k}(x)-\beta_{k}\right)+\sum_{j} \mu_{j} g_{j}(x)
$$

with $\lambda_{k}$ and $\mu_{j}$ to be Lagrangian multipliers, the $\mathrm{K}-\mathrm{T}$ condition is then

$$
\begin{aligned}
\nabla_{x} L(x, \beta) & =\sum_{k} \lambda_{k} \nabla f_{k}(x)+\sum_{j} \mu_{j} \nabla g_{j}(x)=0 \\
\nabla_{\beta_{k}} L(x, \beta) & =w_{k}-\lambda_{k}=0
\end{aligned}
$$

with

$$
\begin{array}{r}
\lambda_{k}\left(f_{k}(x)-\beta_{k}\right)=0 \\
\mu_{j} g_{j}(x)=0 \\
\lambda_{k}, \mu_{j} \geqslant 0
\end{array}
$$

By means of the second equality in (8), we obtain $\lambda_{k}=w_{k} \neq 0$ and its replacement into the first relation of (9) leads to the following relation:

$$
\beta_{k}=f_{k}(x)
$$

This means that all additional constraints related to criteria are active at the optimum. By introducing (10) into (7) and by performing the gradient computation with respect to $x$, we finally get

$$
\begin{aligned}
& \nabla_{x} L(x)=\sum_{k} w_{k} \nabla f_{k}(x)+\sum_{j} \mu_{j} \nabla g_{j}(x)=0 \\
& \mu_{j} g_{j}(x)=0 ; \quad \mu_{j} \geqslant 0
\end{aligned}
$$

Because this is the same optimality condition as (3) for the original weighting problem, formulation (5) is therefore an equivalent form of (2). We can observe that the basic characteristic of the MBF formulation (5) is the transformation of the original weighting objective function, which is linear in the objective space but non-linear and non-monotonic in the design variable space, into a new weighting objective function, which becomes monotonic and linear in the design variable space. In fact, whether the original weighting objective function is linear or non-linear in terms of criteria, the substitution of artificial variables for criteria can be proved to be valid in general sense providing that the original weighting objective function is 
monotonically increased with respect to each criterion. In this case, the solution of the MBF problem is sufficient for convex Pareto optimality.

Finally, it should be indicated that the MBF is also convenient to deal with min-max multicriteria problems where some criteria are themselves identified with the maximization of certain functions, e.g. the stress concentration minimization in structural design:

$$
\begin{gathered}
\operatorname{Min}\left\{\mathbf{f}(x)=\left[f_{1}(x), f_{2}(x), \ldots, f_{r}(x)\right], \max \mathbf{h}(x)=\left\{h_{1}(x), h_{2}(x), \ldots, h_{s}(x)\right\}\right\} \\
g_{j}(x) \leqslant 0, \quad j=1, m
\end{gathered}
$$

This is a non-differentiable problem. In this case, the MBF can be readily established as

$$
\begin{gathered}
\quad \operatorname{Min} \sum_{k=1}^{r+1} w_{k} \beta_{k} \\
f_{k}(x) \leqslant \beta_{k}, \quad k=1, r \\
h_{p}(x) \leqslant \beta_{r+1}, \quad p=1, s \\
g_{j}(x) \leqslant 0, \quad j=1, m
\end{gathered}
$$

with

$$
\sum_{k=1}^{r+1} w_{k}=1, \quad w_{k}>0
$$

\subsection{Relationship between the multibounds formulation and the trade-off method}

The trade-off method also called constraint method is another scalarization approach to solve multicriteria problems. It consists in retaining one of criteria as the objective function and put others as inequality constraints. The original problem (1) is then transformed into the following scalar problem:

$$
\begin{gathered}
\operatorname{Min}_{x} f_{i}(x) \\
f_{k}(x) \leqslant \varepsilon_{k}, \quad k=1, r ; \quad k \neq i \\
g_{j}(x) \leqslant 0, \quad j=1, m
\end{gathered}
$$

where $\varepsilon_{k}$ are artificially imposed upper-bound parameters whose values are prescribed according to the designer's judgement. Theoretically, to reveal the relationship between the MBF and the trade-off method, we can write the related K-T optimality condition of (14) as

$$
\begin{gathered}
\nabla_{x} L(x)=\nabla f_{i}(x)+\sum_{k} \bar{\lambda}_{k} \nabla f_{k}(x)+\sum_{j} \bar{\mu}_{j} \nabla g_{j}(x)=0 \\
\bar{\lambda}_{k}\left(f_{k}(x)-\varepsilon_{k}\right)=0, \quad \bar{\lambda}_{k} \geqslant 0, \quad \bar{\mu}_{j} g_{j}(x)=0, \quad \bar{\mu}_{j} \geqslant 0 \\
k=1, r(k \neq i) ; \quad j=1, m
\end{gathered}
$$

When multiplied by the non-zero value of the weighting coefficient $w_{i}$, we can get

$$
\begin{aligned}
& w_{i} \nabla_{x} L(x)=w_{i} \nabla f_{i}(x)+\sum_{k} w_{i} \bar{\lambda}_{k} \nabla f_{k}(x)+\sum_{j} w_{i} \bar{\mu}_{j} \nabla g_{j}(x)=0 \\
& w_{i} \bar{\lambda}_{k}\left(f_{k}(x)-\varepsilon_{k}\right)=0, \quad w_{i} \bar{\lambda}_{k} \geqslant 0 ; \quad w_{i} \bar{\mu}_{j} g_{j}(x)=0, \quad w_{i} \bar{\mu}_{j} \geqslant 0
\end{aligned}
$$


Now, the comparison of (8), (9) and (16) shows that if the MBF is equivalent to the trade-off method and if both of them provide the same optimum solution, following relations should exist.

For weighting coefficients,

$$
w_{i}=\frac{1}{1+\sum_{k \neq i} \bar{\lambda}_{k}}, \quad w_{k}=w_{i} \bar{\lambda}_{k}=\frac{\bar{\lambda}_{k}}{1+\sum_{k \neq i} \bar{\lambda}_{k}}
$$

and for Lagrangian multipliers,

$$
\lambda_{k}=\frac{w_{i} \bar{\lambda}_{k}}{w_{i}\left(1+\sum_{k \neq i} \bar{\lambda}_{k}\right)}=\frac{\bar{\lambda}_{k}}{1+\sum_{k \neq i} \bar{\lambda}_{k}}, \quad \mu_{j}=\frac{w_{i} \bar{\mu}_{j}}{w_{i}\left(1+\sum_{k \neq i} \bar{\lambda}_{k}\right)}=\frac{\bar{\mu}_{j}}{1+\sum_{k \neq i} \bar{\lambda}_{k}}
$$

From these relations, it can be observed that the weighting coefficients uniquely depend upon the Lagrangian multipliers of the trade-off problem. Therefore, the conversion of the tradeoff problem into MBF problem can be easily performed if the dual approach is adopted to solve (14) because Lagrangian multipliers will be automatically provided as the by-products of solution results. Note that in the work of Das and Dennis [14], a similar relationship as above was elaborated between their normal-boundary intersection subproblem and the goal programming problem.

\section{ADAPTATION OF THE CONLIN OPTIMIZER TO THE RESOLUTION OF THE MULTIBOUNDS PROBLEM}

Firstly, let us have a brief summary of the CONLIN approximation. Details can be found in the work of Fleury [15]. For a given function $f(x)$, the CONLIN approximation at $x=x^{0}$ has the expression

$$
f(x) \approx f\left(x^{0}\right)+\sum_{+} f^{\prime}\left(x^{0}\right)\left(x_{i}-x_{i}^{0}\right)-\sum_{-} f^{\prime}\left(x^{0}\right)\left(x_{i}^{0}\right)^{2}\left(\frac{1}{x_{i}}-\frac{1}{x_{i}^{0}}\right)
$$

with $\sum_{+}$and $\sum_{-}$to be the summation over the terms with positive and negative first-order derivatives, respectively. Three basic characteristics are held for (19):

- Convexity: The dual approach can be efficiently used to solve the optimization subproblem for which the objective function and constraints are explicitly defined by (19).

- Separability of design variables: Therefore, each primal variable $x_{i}$ can be explicitly expressed as a function of dual variables (Lagrangian multipliers).

- Monotonicity: The approximation is hence suitable to describe structural responses such as stresses and displacements in terms of transversal sizing and even shape design variables.

However, as confirmed by the authors with the help of numerical tests, when the CONLIN approximation is directly applied together with the dual approach to solve the original weighting problem (1), the optimization process will surely fail and diverge. The reason is that multiobjective functions are conflicting and incommensurable among them. Hence, the preference weighting objective function will not be monotonic and it will be unsuitable for the CONLIN approximation. 


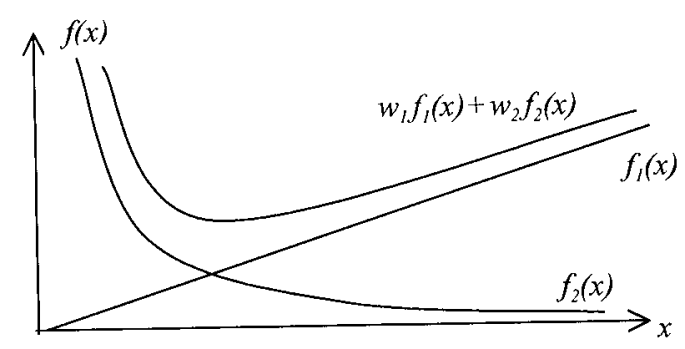

Figure 1. Convex combination of multiobjective functions.

To have a clear idea, imagine for example the simultaneous minimization of a plate structure weight of the mathematical form $f_{1}(x)=a x$ and its flexibility of the form $f_{2}(x)=b / x^{3}$ in terms of the thickness design variable $x$. As shown in Figure 1, the convex combination of these two functions is no longer monotonic.

The proposed MBF formulation is well adapted to the CONLIN approximation because the highly non-linear preference objective function is replaced by a linear function and that each criterion can be individually handled in the standard CONLIN form as for one criterion optimization problem. At the initial design point, the explicit subproblem is then constructed in the form

$$
\begin{gathered}
\operatorname{Min} \sum_{k} w_{k} \beta_{k} \\
a_{k}^{0}+\sum_{i}^{+} a_{i k} x_{i}+\sum_{i}^{-} \frac{b_{i k}}{x_{i}}+\frac{c_{k}}{\beta_{k}} \leqslant 0, \quad k=1, r \\
d_{j}^{0}+\sum_{i}^{+} d_{i j} x_{i}+\sum_{i}^{-} \frac{e_{i j}}{x_{i}} \leqslant 0, \quad j=1, m
\end{gathered}
$$

with $x_{i}$ and $\beta_{k}$ to be the design variables. The coefficients $a_{k}^{0}, a_{i k}, b_{i k}, c_{k}, d_{j}^{0}, d_{i j}$ and $e_{i j}$ to be all constants depending upon the function values and the first-order derivatives at the initial design point. Note that the reciprocal term is used for $\beta_{k}$ in (20) because the related first-order derivative is negative and equal to -1 . To avoid the singularity of this term, $\beta_{k}$ should be limited to take the positive value. This limitation can be realized through the conventional translation shift for each criterion.

It is important to remark that the basic purpose of the MBF method is devised to be a general formulation well adapted to the numerical solution procedure of any available optimizers in use. Its junction with the CONLIN optimizer is just one efficient combination of all. The reason to favour this combination is based on the fact that convex programming methods have been appreciated for about 20 years in amounts of numerical practices and they acquire great successes both in suitability and in efficiency for structural optimization. One can see in the next section that these successes can equally be met with in multicriteria optimization thanks to the MBF formulation.

\section{NUMERICAL EXAMPLES}

Shell elements are widely used in the sizing optimization of complex structures. Two shell panels are studied here to test the efficiency of the MBF method and the applicability of 


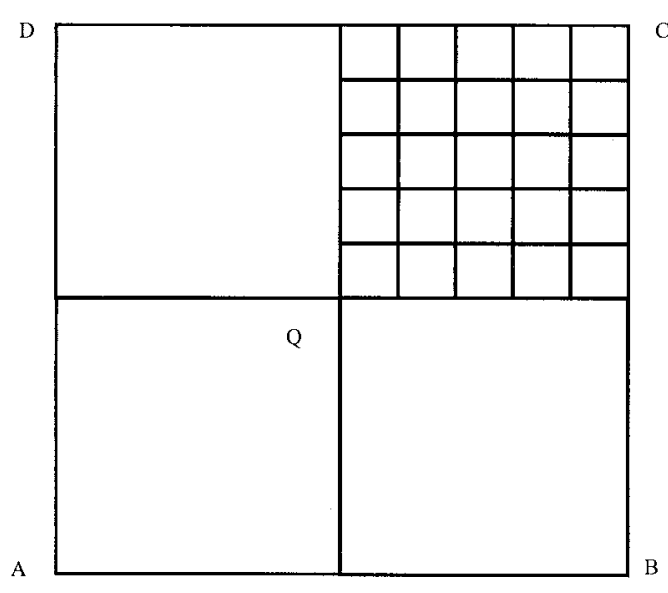

Figure 2. Plane shell panel problem.

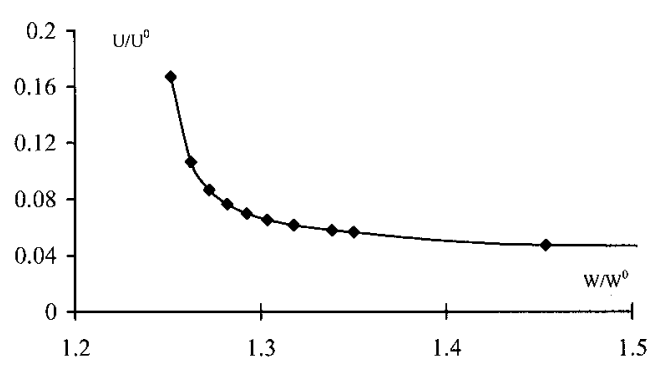

Figure 3. Pareto-optimal curve for weightdisplacement minimization (first case).

CONLIN algorithm in multicriteria optimization. The convergence history of the objective function defined in MBF and the Pareto-optimal curve will be examined. The design tool is integrated with the sensitivity analysis procedure developed in the framework of ABAQUS optimization system by Zhang et al. [16]. Two cases are taken into account:

(1) The simultaneous minimization of the panel weight and the transversal point-wise displacement at the central point of the panel. Constraints are imposed on the element thickness. The mathematical programming statement is

$$
\begin{gathered}
\operatorname{Min} w_{1}\left(\frac{W}{W^{0}}\right)+w_{2}\left(\frac{U}{U^{0}}\right) \\
\underline{t}_{i} \leqslant t_{i} \leqslant \bar{t}_{i}
\end{gathered}
$$

(2) The simultaneous minimization of the panel weight and the stress concentration in the panel.

$$
\operatorname{Min}\left[w_{1}\left(\frac{W}{W^{0}}\right)+w_{2} \max _{i}\left(\frac{\sigma_{i \mathrm{v} . \mathrm{m}}}{\sigma_{\max }^{0}}\right)\right]
$$

In both cases, each criterion is normalized to be dimensionless by its initial value. Besides, the weighting coefficients are uniformly discretized as $w_{1}=[0,0.1,0.2, \ldots, 0.9,1]$ and $w_{2}=[1,0.9$, $0.8, \ldots, 0.1,0]$.

\subsection{Square plane shell panel}

The first structure shown in Figure 2 is a panel loaded by a transversal force $F$ at the central point $Q$. Four edges are completely clamped. Due to the symmetry, only a quarter of the panel will be considered. A discretization of $5 \times 5$ finite element mesh is used by means 


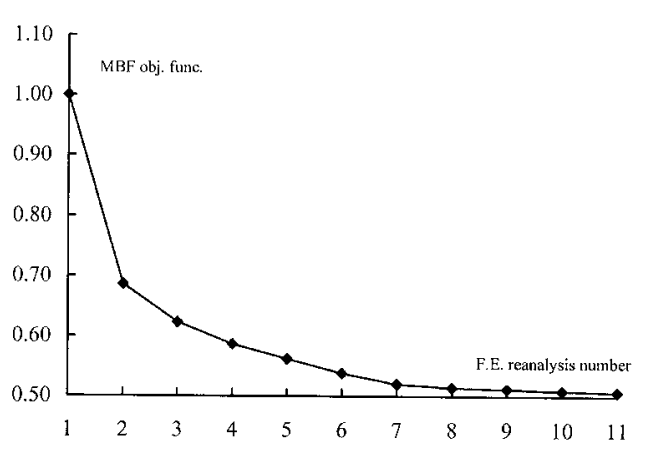

Figure 4. Iteration history of the MBF objective function for weight-displacement minimization $\left(w_{1}=w_{2}=0.5\right)$.

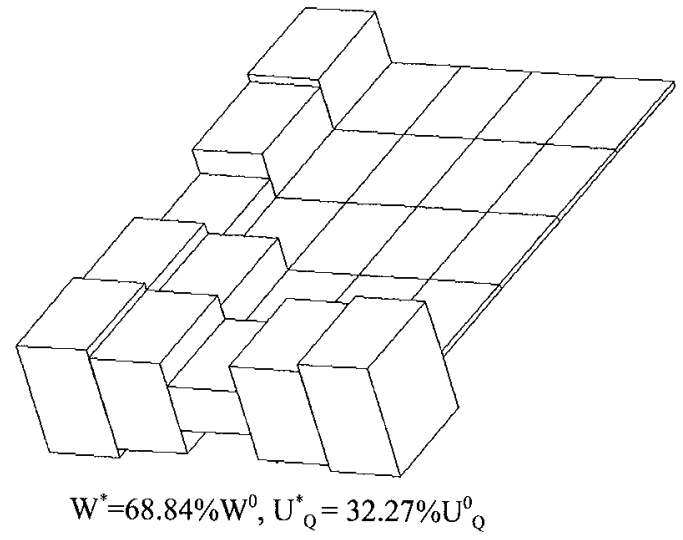

Figure 5. Thickness distribution in weightdisplacement minimization $\left(w_{1}=w_{2}=0.5\right)$.

of the four-node S4R shell elements of the ABAQUS library. Thickness of each element is considered as one design variable. Initial data are given below.

$A B=B C=20$ in, Initial thickness: $t^{0}=1$ in

Lower bound: $\underline{t}=0.1$ in, Upper bound: $\bar{t}=2$ in

Physical properties: $E=10^{7} \mathrm{psi}, v=0.3, \rho=0.1 \mathrm{lb} / \mathrm{in}^{3}$

Loads: $F=4 \times 10^{5} \mathrm{lb}$

The initial design corresponds to the weight of the studied portion of the panel $W^{0}=10 \mathrm{lb}$. The transversal displacement $U_{Q}^{0}=1.061$ in at the point $Q$; the maximum Von-Mises stress $\sigma_{\mathrm{v} . \mathrm{m}}^{0}=4.2 \times 10^{5} \mathrm{psi}$.

In the first case, the criterion associated with the displacement minimization is, in fact, equivalent to minimizing the compliance of the panel due to the concentrated force $F$. For different discrete values of weighting coefficients, the problem will be repeatedly solved by using the MBF formulation in junction with the CONLIN optimizer. The Pareto-optimal curve is then obtained and plotted in Figure 3. Note that the real extremity point in the right-hand of the curve, which corresponds to the solution of the minimum of the compliance and the maximal thickness of all elements is not plotted since it is located far from the zoom of the actual curve segment. In this case study, numerical results point out that the iteration history is indeed stable and has a quick convergence rate for all discrete values of weighting coefficients given above. For example, the iteration curve shown in Figure 4 indicates that the convergence of MBF objective function with the weighting coefficients $w_{1}=w_{2}=0.5$ has nearly no significant variation after 10 finite element analyses. The related optimal thickness distribution and Von-Mises stresses are also shown in Figures 5 and 6, respectively.

Similarly, in the second case of minimizing the panel weight and stress concentration, it is found that the MBF method and the CONLIN algorithm are also well adapted to 


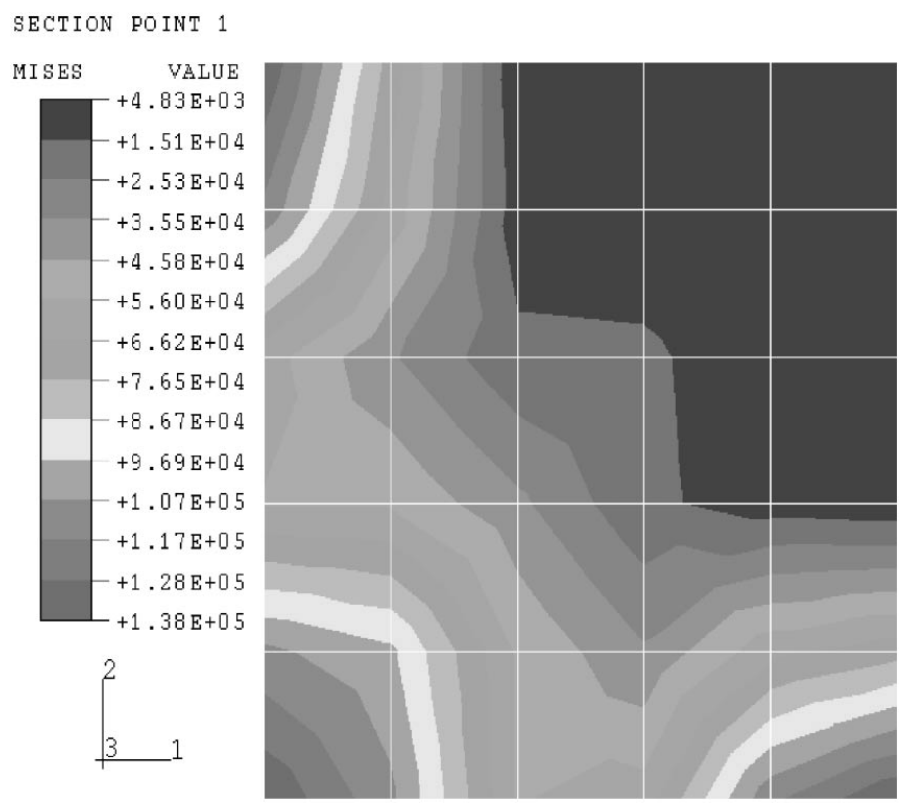

Figure 6. Von-Mises stress distribution in weight-displacement minimization $\left(w_{1}=w_{2}=0.5\right)$.

all given weighting coefficient values. Take, for example, the case $w_{1}=w_{2}=0.5$, the optimal thickness distribution and Von-Mises stresses are shown in Figures 7 and 8, respectively. The convergence curve is shown in Figure 9. It can be seen that the layout of materials is concentrated along the symmetric lines of the panel, where maximal constraints occur.

\subsection{Curved shell panel}

The second problem is given in Figure 10. Two edges AB and CD are clamped. The shell is loaded by a concentrated load $F_{z}=4 \times 10^{4} \mathrm{lb}$ in the transversal direction. Due to the symmetry, only a quarter of the whole panel needs to be considered. The finite element model corresponds to a mesh of $18 \times 18$ S4R shell elements of ABAQUS library. By grouping each set of 9 finite elements, a total number of 36 independent thickness design variables are then defined together with 2 artificial design variables associated with the bi-criteria. For this problem, because of the ratio of initial shell thickness to the radius: $t^{0} / R=3 \times 10^{-3} \ll 1$, the hypothesis of the thin shell elements is assumed. Initial data are given below.

Radius: $R=300$ in, Length: $A B=C D=600$ in, Open angle: $\theta=80^{\circ}$

Initial thickness: $t^{0}=1 \mathrm{in}$, Lower bound: $\underline{t}=0.01 \mathrm{in}$, Upper bound: $\bar{t}=4$ in

Physical properties: $E=10^{7} \mathrm{psi}, v=0.3, \rho=0.1 \mathrm{lb} / \mathrm{in}^{3}$

Stress limit: $\sigma_{\mathrm{v} . \mathrm{m}} \leqslant 25000 \mathrm{psi}$ 


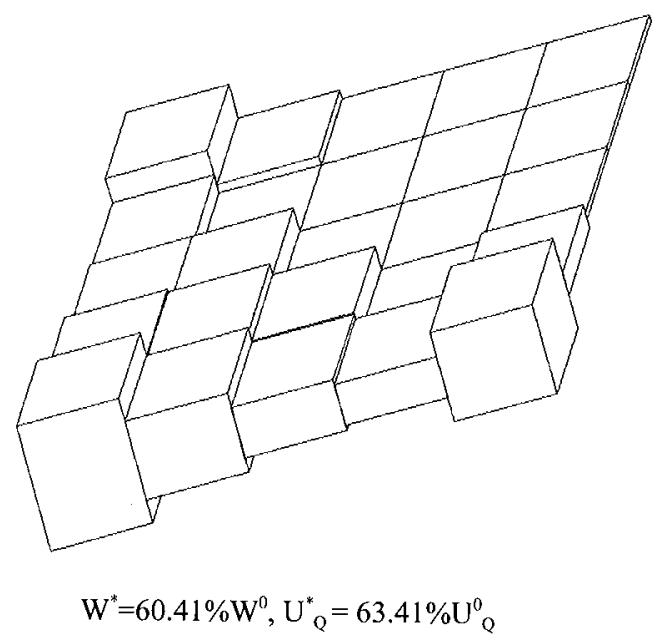

Figure 7. Thickness distribution in weight-stress minimization $\left(w_{1}=w_{2}=0.5\right)$.

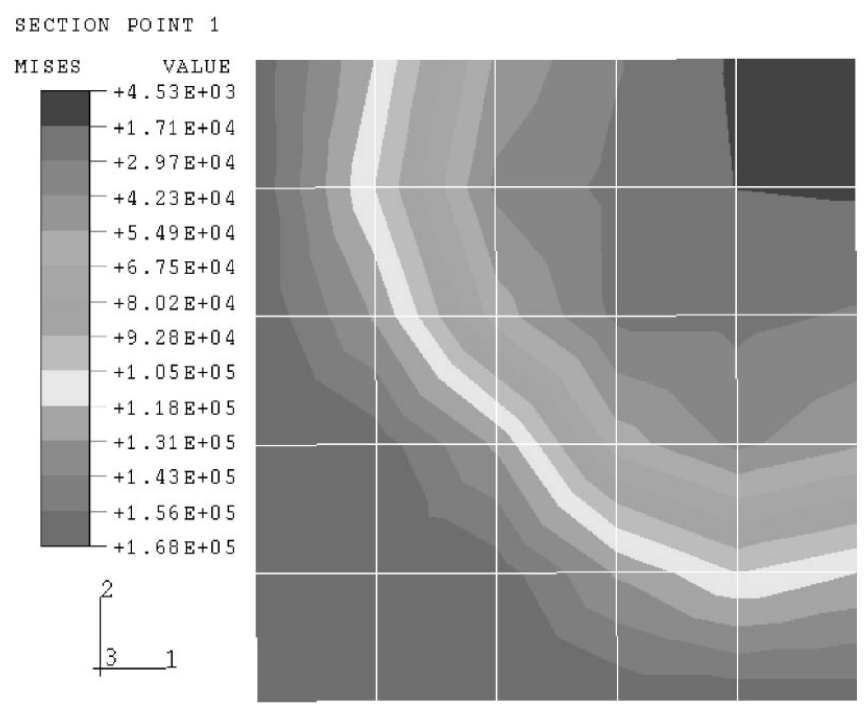

Figure 8. Von-Mises stress distribution in weight-stress minimization $\left(w_{1}=w_{2}=0.5\right)$.

In the first case of weight-displacement minimization where the transversal displacement of the central point of the entire panel is concerned, the Pareto-optimal solution curve is obtained and given in Figure 11. For the second case of weight-stress minimization with weighting coefficients $w_{1}=w_{2}=0.5$, results of final thickness and Von-Mises stress distributions are plotted in Figures. 12 and 13, the iteration history is shown in Figure 14. The final weight $W^{*}$ is reduced and equal to 82.04 per cent $W^{0}$ with respect to the initial weight; the maximum 


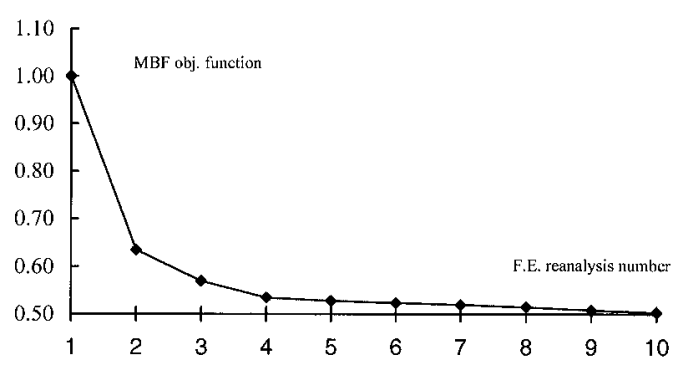

Figure 9. Iteration history of the MBF objective function for weight-stress minimization $\left(w_{1}=w_{2}=0.5\right)$.

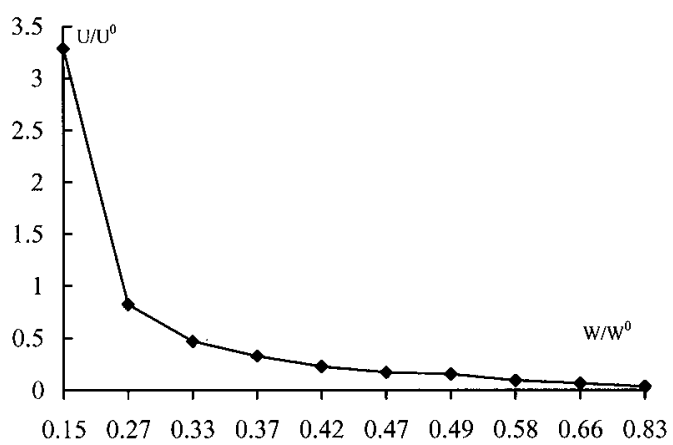

Figure 11. Pareto-optimal curve for weight and displacement minimization (first case).

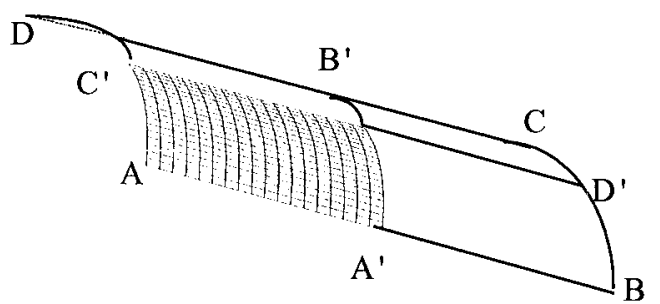

Figure 10. Curved shell panel problem.

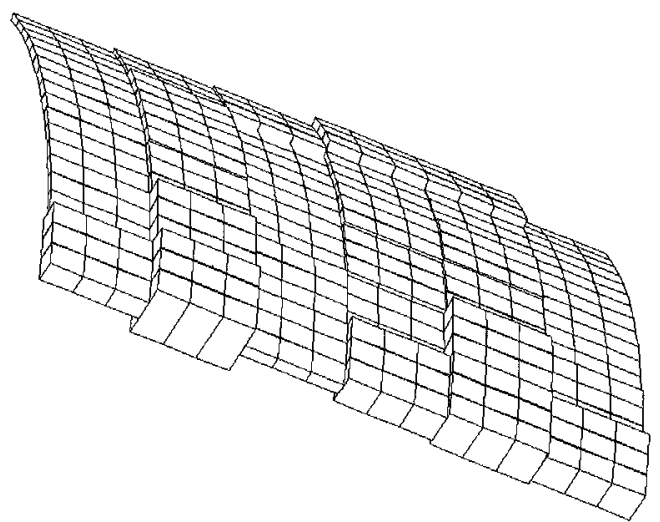

Figure 12. Thickness distribution in weight-stress minimization $\left(w_{1}=w_{2}=0.5\right)$.

Von-Mises stress $\sigma_{\mathrm{v} . \mathrm{m}}^{*}$ is also reduced with respect to the initial maximum Von-Mises stress and equal to 44.74 per cent $\sigma_{\mathrm{v} . \mathrm{m}}^{0}$.

\section{CONCLUSIONS}

A multibounds formulation (MBF) is presented for multicriteria structural optimization. Although the mathematical proof shows that this formulation is intrinsically equivalent to the classical weighting formulation, its simplified form is suitable to the numerical resolution procedure. The MBF formulation readily extends the one criterion problem-oriented application of convex programming methods, e.g. the CONLIN optimizer and its variants to multicriteria optimization. In addition, the MBF formulation is shown to be a unified formulation of one criterion and multicriteria problems. Numerical results of shell design problems indicate that the integration of the MBF formulation with the CONLIN optimizer constitutes an applicable and efficient structural optimization tool. The iteration history is found to be stable and 


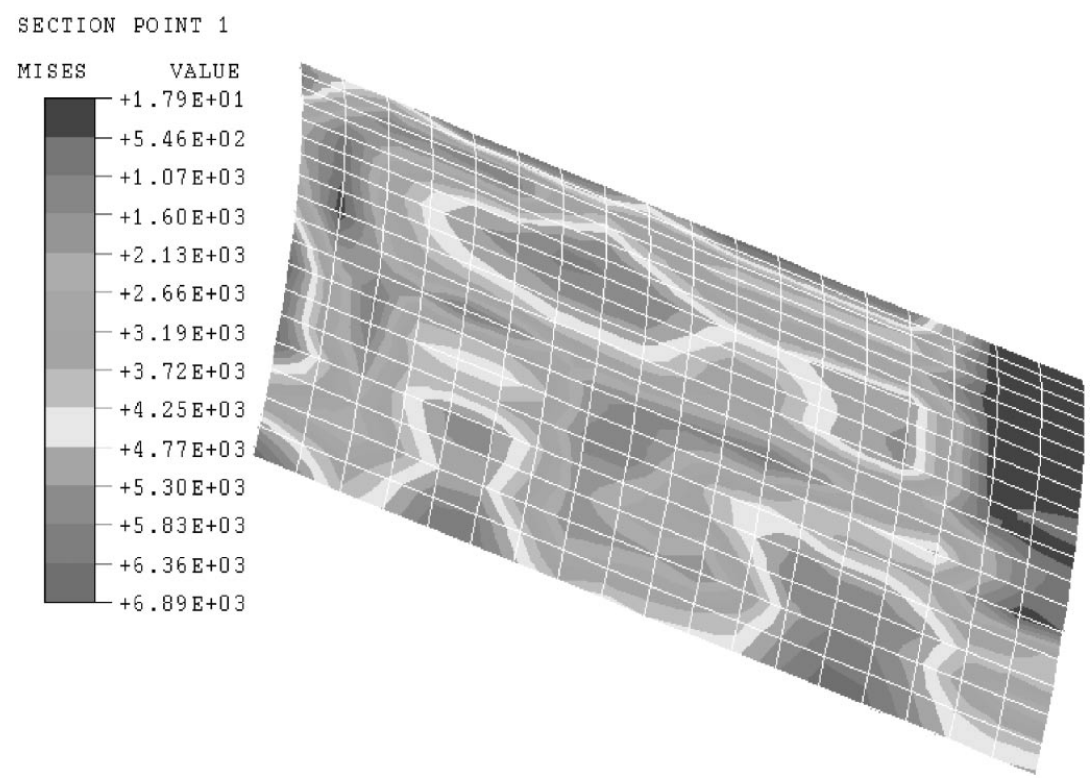

Figure 13. Von-Mises stress distribution in weight-stress minimization $\left(w_{1}=w_{2}=0.5\right)$.

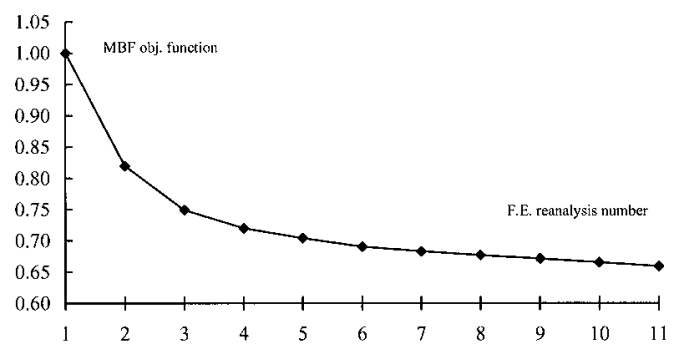

Figure 14. Iteration history of the MBF objective function for weight-stress minimization.

very satisfactory. The convergence is not sensible to the selections of weighting coefficient values.

\section{ACKNOWLEDGEMENTS}

This work was supported by the Aeronautical Foundation (00B53005) of China within the scope of Cheung Kong Scholars Programme.

\section{REFERENCES}

1. Eschnauer H, Koski J, Osyczka A. Multicriteria Design Optimization. Springer: Berlin, Heidelberg, New York, 1990.

2. Stadler W. Multicriteria Optimization in Engineering and in Sciences. Plenum Press: New York, 1988. 
3. Osyczka A. Multicriterion Optimization in Engineering. Chichester: Ellis Horwood, 1978.

4. Saravanos DA, Chamis CC. Multiobjective shape and material optimization of composite structures including damping. AIAA Journal 1992; 30(3):805-813.

5. Schy AA, Giesy DP, Johnson KG. Pareto-optimal multi-objective design of airplane control systems. Proceedings of the 1980 Joint Automatic Control Conference. ASME, New York, 1980; WP1-A.

6. Das I, Dennis JE. A closer look at drawbacks of minimizing weighted sums of objectives for Pareto set generation in multicriteria optimization problems. Structural Optimization 1997; 14:63-69.

7. Li YS, Fadel GM, Wiecek MM. Approximating Pareto curves using the hyper-ellipse. ISSMO/NASA/AIAA First Internet Conference on Approximation and Fast Reanalysis Techniques in Engineering Optimisation, 1999.

8. Pietrzak J. A systematic search for Pareto optimum solutions. Structural Optimization 1999; 17:79-81.

9. Grandhi RV, Bharatram G. Compound scaling algorithm for multiobjective functions. Microcomputers in Civil Engineering 1995; 10:281-289.

10. Koski J. Defectiveness of weighting method in multicriterion optimization of structures. Communications in Applied Numerical Methods 1985; 1:333-337.

11. Lin JG. Multiple-objective problems: Pareto-optimal solutions by method of proper equality constraints. IEEE Transactions on Automatic Control AC-21 1976; 641-650.

12. Bendsøe MP, Olhoff N, Taylor JE. A variational formulation for multicriteria structural optimization. Journal of Structural Mechanics 1983; 11:523-544.

13. Olhoff N. Multicriterion structural optimization via bound formulation and mathematical programming. Structural Optimization 1989; 1:11-17.

14. Das I, Dennis JE. Normal-boundary intersection: a new method for generating Pareto optimal points in nonlinear multicriteria optimization problems. http://www.owlnet.rice.edu/ indra/NBI homepage.html 1997.

15. Fleury C. First and second order convex approximation strategies in structural optimization. Structural Optimization 1989; 1:3-10.

16. Zhang WH, Domaszewski M, Bassir H. Developments of sizing sensitivity analysis with the ABAQUS code. Structural Optimization 1999; 17:219-225. 